\title{
Neuroinflammation in Alzheimer's disease wanes with age
}

\author{
Jeroen JM Hoozemans ${ }^{1 *}$, Annemieke JM Rozemuller ${ }^{1}$, Elise S van Haastert ${ }^{1}$, Piet Eikelenboom ${ }^{2,3}$ and \\ Willem A van Gool ${ }^{3}$
}

\begin{abstract}
Background: Inflammation is a prominent feature in Alzheimer's disease (AD). It has been proposed that aging has an effect on the function of inflammation in the brain, thereby contributing to the development of age-related diseases like AD. However, the age-dependent relationship between inflammation and clinical phenotype of AD has never been investigated.

Methods: In this study we have analysed features of the neuroinflammatory response in clinically and pathologically confirmed AD and control cases in relation to age (range 52-97 years). The mid-temporal cortex of 19 controls and 19 AD cases was assessed for the occurrence of microglia and astrocytes by immunohistochemistry using antibodies directed against CD68 (KP1), HLA class II (CR3/43) and glial fibrillary acidic protein (GFAP).

Results: By measuring the area density of immunoreactivity we found significantly more microglia and astrocytes in $A D$ cases younger than 80 years compared to older AD patients. In addition, the presence of KP1, CR3/43 and GFAP decreases significantly with increasing age in AD.

Conclusion: Our data suggest that the association between neuroinflammation and AD is stronger in relatively young patients than in the oldest patients. This age-dependent relationship between inflammation and clinical phenotype of AD has implications for the interpretation of biomarkers and treatment of the disease.
\end{abstract}

Keywords: Alzheimer?'?s disease, microglia, astrocyte, aging

\section{Background}

Alzheimer's disease (AD) is a chronic neurodegenerative disease and is the most common cause of dementia. Two hallmarks of the disease are senile plaques, which are mainly composed of extracellular deposits of amyloid $\beta$ $(\mathrm{A} \beta)$, and neurofibrillary tangles, which consist of intracellular aggregates of aberrantly phosphorylated tau protein. Senile plaques are associated with an inflammatory response as shown by an increased presence of activated complement proteins, cytokines, and activated microglia and astrocytes [1]. It is suggested that this inflammatory response, also referred to as neuroinflammation, plays a prominent and early role in AD [2-4]. A role for inflammation in AD has recently gained strong support from

\footnotetext{
* Correspondence: jjm.hoozemans@vumc.nl

'Department of Pathology, VU University Medical Center, P.O. Box 7057,

1007 MB Amsterdam, The Netherlands

Full list of author information is available at the end of the article
}

genome-wide association studies that have identified genes involved in inflammation that are associated with increased risk of developing $\mathrm{AD}[5,6]$.

The inflammatory response in AD is a double-edged sword. It is a self-defence reaction aimed at eliminating injurious stimuli and restoring tissue integrity. However, inflammation may become a harmful process when it becomes chronic. Chronic activation of the inflammatory response in $\mathrm{AD}$ produces pro-inflammatory cytokines, prostaglandins and reactive oxygen species that exacerbate $\mathrm{A} \beta$ deposition and induce neuronal dysfunction [7]. Despite wide acceptance of the idea that inflammation contributes to $\mathrm{AD}$, it remains unclear at what stages of $\mathrm{AD}$ inflammation is beneficial or detrimental [8].

Clinicopathological studies suggest that neuroinflammation, and in particular microglial activation, is an early event in $\mathrm{AD}$ pathology. The volume of tissue occupied by microglia, the brain resident macrophages, increases with
C Biomed Central 
severity of dementia, but peaks in moderately affected cases [2]. The volume density of microglia is already increased in early pathological stages of $\mathrm{AD}$ and in cognitively normal subjects with frequent presence of plaques and tangles [3,4]. Clinical studies using positron emission tomography and the peripheral benzodiazepine ligand PK11195 as a marker for activated microglia indicate that activation of microglia occurs already in mild and early forms of AD-type dementia and precedes cerebral atrophy in $\mathrm{AD}[9,10]$. These data indicate that neuroinflammation is involved at an early stage of AD pathogenesis.

It has recently been proposed that aging has an effect on the function of inflammation in the brain. Some studies have suggested that aging facilitates an imbalance between pro- and anti-inflammatory mechanisms resulting in a low grade, chronic pro-inflammatory status, referred to as inflammaging, while other studies have proposed that microglia deteriorate during aging $[11,12]$. These studies have suggested that the effect of aging on inflammation in the brain contributes to the development of age-related diseases like AD. However, the age-dependent relationship between inflammation and clinical phenotype of AD has never been investigated. In this study, we examined the presence of microglia and astrocytes, as markers of neuroinflammation, in clinically and pathologically confirmed $\mathrm{AD}$ and non-demented control cases in relation to age. Our data suggest that the association between neuroinflammation and $\mathrm{AD}$ is much stronger in relatively young patients as compared to the oldest patients.

\section{Methods \\ Case selection}

Post-mortem brain material was obtained from the Netherlands Brain Bank (Amsterdam, The Netherlands). All donors or their next of kin provided written informed consent for brain autopsy and use of tissue and medical records for research purposes. Between 1999 and 2001 non-demented control and AD cases were sequentially selected. Dementia status at death was determined on the basis of all information available for each case during the last year of life. Severity of dementia was measured using the Global Deterioration Scale (GDS) [13]. Neuropathological evaluation was performed on formalin fixed, paraffin embedded tissue from different sites, including the frontal cortex (F2), temporal pole cortex, parietal cortex (superior and inferior lobule), occipital pole cortex and the hippocampus (essentially CA1 and entorhinal area of the parahippocampal gyrus). The distribution and the density of neurofibrillary tangles was determined in Bodian-stained sections, while senile plaques were stained with the methenamine silver method [14]. AD pathology was staged according to Braak and Braak [15]. Cases with mixed Parkinson's or Lewy body disease were excluded from the study. Demographic information, clinical status, neuropathological staging and APOE genotype are shown in Table 1.

\section{Immunocytochemistry}

For immunohistochemical staining, formalin fixed (4\%, 24 h) paraffin embedded tissue from mid-temporal cortex was used. Sections ( $5 \mu \mathrm{m}$ thick) were mounted on superfrost-plus tissue slides (Menzel-Gläser, Germany) and deparaffinized. Subsequently, sections were immersed in $0.3 \% \mathrm{H}_{2} \mathrm{O}_{2}$ in methanol for $30 \mathrm{~min}$ to quench endogenous peroxidase activity, and treated in $10 \mathrm{mM} \mathrm{pH} 6.0$ citrate buffer heated by microwave during $10 \mathrm{~min}$ for antigen retrieval. Normal serum and antibodies were dissolved in phosphate-buffered saline (PBS) containing 1\% (w/v) bovine serum albumin (BSA, Boehringer Mannheim, Germany). Sections were pre-incubated for $10 \mathrm{~min}$ with normal rabbit serum (DAKO, Glostrup, Denmark). Primary antibodies were incubated for $1 \mathrm{hr}$ at room temperature. See Table 2 for antigen, dilution and source of primary antibodies. After washing with PBS, slides were incubated with biotin-conjugated rabbit anti-mouse antibody (rabbit anti-mouse $\mathrm{F}(\mathrm{ab}$ ')2, 1:500 dilution, DAKO) for $30 \mathrm{~min}$. Subsequently, slides were incubated with streptavidin-biotin horseradish peroxidase complex (streptABComplex/ HRP, 1:200 dilution, DAKO) for $60 \mathrm{~min}$. Color was developed using 3,3'-diaminobenzidine $(0.1 \mathrm{mg} / \mathrm{ml}, 0.02 \%$ $\mathrm{H} 2 \mathrm{O} 2,3 \mathrm{~min})$ as chromogen. Sections were mounted with Entellan (Merck, Darmstadt, Germany).

\section{Quantitative analyses}

For each case an area between the top and the depth of a gyrus was selected at random. Contiguous microscopic fields from the pial surface to the boundary with white matter perpendicularly to the axis of a gyrus made up a column. At least three columns were assessed per individual case. AT8-immunoreactive neurofibrillary tangles were counted using a $40 \times$ objective $\left(0.16 \mathrm{~mm}^{2}\right)$. A $\beta$ - and AT8positive plaques were counted using a $10 \times$ objective $(0.64$ $\mathrm{mm}^{2}$ ). In order to measure the amount of immunoreactivity for KP1, CR3/43 and GFAP, the area density of immunoreactivity was measured [4]. Contiguous microscopic fields arranged in columns were examined with a $10 \times$ objective. Full color images were obtained using a Zeiss light microscope equipped with a digital camera. The area density was quantified using Image-Pro Plus analysis software (Media Cybernetics, Silver Spring, MD). Using this method the percentage of the area of interest that is immunoreactive for a specific antibody is measured. Assessments for different antibodies were performed in adjacent sections and blind to the pathological and clinical categorization.

\section{Statistical analyses}

The Kruskall-Wallis test was used to evaluate differences between groups followed by the Mann-Whitney U 
Table 1 Clinical status, demographic information, disease duration, neuropathological staging and APOE genotype of control and AD cases used in this study.

\begin{tabular}{|c|c|c|c|c|c|c|c|c|c|}
\hline Case & Diagnosis & Sex & Age (years) & Duration (years) & GDS & Braak & Brain weight (grs) & PMD (hours: minutes) & APOE genotype \\
\hline 1 & CTRL & $\mathrm{F}$ & 52 & & 0 & 0 & 1268 & $6: 50$ & $3-3$ \\
\hline 2 & CTRL & M & 53 & & 0 & 0 & 1341 & $14: 25$ & $3-3$ \\
\hline 3 & CTRL & $\mathrm{F}$ & 64 & & 0 & 0 & 1169 & $8: 35$ & $4-2$ \\
\hline 4 & CTRL & $M$ & 70 & & 0 & 0 & 1560 & $7: 45$ & $4-3$ \\
\hline 5 & CTRL & $\mathrm{F}$ & 72 & & 0 & 1 & 1296 & $6: 45$ & $4-3$ \\
\hline 6 & CTRL & $M$ & 73 & & 0 & 0 & 1267 & $24: 45$ & $3-3$ \\
\hline 7 & CTRL & $\mathrm{F}$ & 76 & & 0 & 1 & 1212 & $4: 50$ & $3-2$ \\
\hline 8 & CTRL & $\mathrm{F}$ & 77 & & 0 & 2 & 1213 & 9:15 & 4-3 \\
\hline 9 & CTRL & $M$ & 78 & & 0 & 1 & 1467 & $5: 35$ & $4-3$ \\
\hline 10 & CTRL & $F$ & 78 & & 0 & 1 & 1047 & $6: 30$ & $4-3$ \\
\hline 11 & CTRL & $M$ & 78 & & 0 & 1 & 1332 & $6: 55$ & $3-3$ \\
\hline 12 & CTRL & $M$ & 79 & & 0 & 1 & 1334 & $7: 40$ & $4-3$ \\
\hline 13 & CTRL & $M$ & 82 & & 0 & 2 & 1468 & $13: 35$ & $3-3$ \\
\hline 14 & CTRL & $F$ & 82 & & 0 & 1 & 1280 & $5: 30$ & $3-2$ \\
\hline 15 & CTRL & $\mathrm{F}$ & 83 & & 0 & 2 & 1102 & $7: 45$ & 3-3 \\
\hline 16 & CTRL & $\mathrm{F}$ & 85 & & 0 & 3 & 1199 & 9:00 & $4-3$ \\
\hline 17 & CTRL & $\mathrm{F}$ & 88 & & 0 & 2 & 1152 & $5: 40$ & 3-3 \\
\hline 18 & CTRL & $F$ & 92 & & 0 & 1 & 1031 & $7: 15$ & $3-2$ \\
\hline 19 & CTRL & $M$ & 98 & & 0 & 1 & 1203 & $8: 40$ & $3-3$ \\
\hline 20 & $A D$ & $F$ & 65 & 16 & 7 & 6 & 1115 & $5: 40$ & $3-3$ \\
\hline 21 & $A D$ & $M$ & 67 & $?$ & 6 & 5 & 1170 & $5: 22$ & $4-4$ \\
\hline 22 & $A D$ & $\mathrm{~F}$ & 70 & 10 & 7 & 6 & 1020 & 4:30 & 3-3 \\
\hline 23 & $A D$ & $\mathrm{~F}$ & 76 & 12 & 7 & 6 & 1170 & $5: 30$ & $4-3$ \\
\hline 24 & $A D$ & $M$ & 78 & 8 & 6 & 5 & 1298 & $7: 45$ & $4-4$ \\
\hline 25 & $A D$ & $F$ & 80 & $?$ & 7 & 6 & 946 & $5: 20$ & $4-4$ \\
\hline 26 & $A D$ & $M$ & 80 & 9 & 5 & 6 & 1240 & $4: 20$ & $4-3$ \\
\hline 27 & $A D$ & $F$ & 82 & 7 & 7 & 5 & 1013 & $4: 10$ & $4-3$ \\
\hline 28 & $A D$ & $\mathrm{~F}$ & 83 & 5 & 5 & 4 & 1288 & $5: 20$ & 4-3 \\
\hline 29 & $A D$ & $F$ & 83 & 3 & 6 & 5 & 1095 & $3: 45$ & $4-3$ \\
\hline 30 & $A D$ & $F$ & 86 & 10 & 6 & 4 & 1158 & 4:45 & $4-3$ \\
\hline 31 & $A D$ & $F$ & 87 & 2 & 7 & 3 & 976 & $3: 55$ & $4-2$ \\
\hline 32 & $A D$ & $\mathrm{~F}$ & 89 & 2 & 5 & 3 & 1212 & $4: 10$ & 3-3 \\
\hline 33 & $A D$ & $F$ & 91 & 4 & 4 & 3 & 1161 & $9: 35$ & $4-3$ \\
\hline 34 & $A D$ & $\mathrm{~F}$ & 91 & 7 & 5 & 4 & 1101 & $3: 45$ & $4-3$ \\
\hline 35 & $A D$ & $\mathrm{~F}$ & 93 & 10 & 6 & 4 & 943 & $6: 15$ & $3-3$ \\
\hline 36 & $A D$ & $\mathrm{~F}$ & 94 & 4 & 5 & 2 & 1050 & $3: 15$ & 3-3 \\
\hline 37 & $A D$ & $F$ & 94 & 7 & 6 & 2 & 987 & $6: 35$ & $3-3$ \\
\hline 38 & $A D$ & $F$ & 97 & 7 & 7 & 3 & 1016 & $7: 00$ & $3-3$ \\
\hline
\end{tabular}

CTRL, control case; AD, Alzheimer's disease patient; F, female; M, male; GDS, Global Deterioration Scale; gr, gram; PMD, post-mortem delay.

Table 2 Primary antibodies used in this study.

\begin{tabular}{lllll}
\hline Antibody & Species & Antigen & Dilution & Source \\
\hline KP1 & mouse & CD68 & $1: 400$ & Dako, Glostrup, Denmark \\
\hline CR3/43 & mouse & HLA-DP/DQ/DR & $1: 50$ & Dako, Glostrup, Denmark \\
\hline GFAP (clone 6F2) & mouse & GFAP & $1: 10$ & Monosan, Uden, The Netherlands \\
\hline AT8 & mouse & Tau pSer202 and pThr205 & $1: 1000$ & Pierce, Rockford, IL, USA \\
\hline A 1 1-17 & mouse & A 1 1-17 & $1: 50$ & Dako, Glostrup, Denmark \\
\hline
\end{tabular}


test, to test differences between pairs of groups. Linear regression analysis was performed to model the relation between age and different variables in controls and AD cases. Beta coefficients of the relation between age and different variables were compared between control and AD cases. A p value $<0.05$ was taken as significant.

\section{Results}

The relative occurrence of AD pathological hallmarks and neuroinflammation was assessed in two age-groups. A post-mortem age of 80 years was used as a cut-off as it provided comparable group sizes. The number of $A \beta$ immunoreactive plaques and AT8-positive plaques and tangles were determined in each case. For assessment of KP1, CR3/43 and GFAP immunoreactivity, the area density was determined and expressed as the percentage of area positive for each specific marker (Figure 1). For all markers there were significant differences between $\mathrm{AD}$ and control cases in the group of 80 years and younger, as well in the group older than 80 years. Except for CR3/43, no significant difference between control and AD cases could be observed in the group older than 80 years. Within the AD group, significant differences in AT8-positive tangles, KP1 and GFAP were observed between AD cases older and younger than 80 years. This data indicates that, in contrast to $A \beta$ deposits and AT 8 positive plaques, the occurrence of tangles, microglia and astrocytes is lower in old AD cases (> 80 years) compared to younger AD cases.

To study more directly the association between age of death and the area density of KP1, CR3/43 and GFAP in $\mathrm{AD}$ cases and controls, regression analysis was performed. The occurrence of KP1, CR3/43 and GFAP rapidly decreases with age in AD cases, in contrast to control cases (Figure 2). For all markers the 95\% confidence intervals of control and AD cases start to overlap between post-mortem ages of 85 and 90 years. A significant beta-coefficient for regression over age was observed for KP1, CR3/43 and GFAP in AD cases. Comparison of respective beta-coefficients for regression over age confirmed significant differences between AD and control cases for KP1, CR3/43 and GFAP (Table 3). These data indicate that the occurrence of microglia and astrocytes decreases over age in $\mathrm{AD}$, in contrast to control cases, suggesting that the association between neuroinflammation and AD is stronger in cases with relatively young age.

Apolipoprotein $\mathrm{E}$ (apoE) has been shown to play a role in the innate immune response, and inheritance of the APOE4 allele is associated with increased risk of developing AD $[16,17]$. The APOE genotype is indicated in Table 1 . In this study, the incidence of APOE4 in control cases younger than 80 years is $29 \%$ compared to $7 \%$ in older control cases. For AD cases, the incidence of APOE4 in cases younger than 80 years is $57 \%$ compared to $29 \%$ in older AD cases.

\section{Discussion}

In this study, we compared the age-dependent presence of microglia and astrocytes, which is indicative of a neuroinflammatory response, in controls and AD cases. We show that the association between neuroinflammation and AD is stronger in relatively young AD cases compared to old AD cases. The difference in occurrence of these neuroinflammatory markers between AD and control cases decreases over age. Non-demented controls and AD cases were selected from sequentially performed autopsies over a period of two years. Inclusion criteria for controls included no reported signs of cognitive impairment during life. The presence of neurofibrillary tangles or neuritic plaques after neuropathological examination was not used as an exclusion criteria for controls (see Braak stage, Table $1)$. Inclusion criteria for $A D$ cases were based on clinical signs of AD-type dementia. Dementia status at death was determined on the basis of all information available for each case during the last year of life. Severity of dementia was measured using the Global Deterioration Scale (GDS), which encompasses activities of daily living, behaviour and cognition, and is not affected by the educational level of patients [13]. In using the above mentioned inclusion criteria and sequential selection over a period of two years we sought to avoid possible bias related to age and severity of the underlying pathology that causes the disease.

Previous reports have indicated that activation or presence of microglia as well as the occurrence of gliosis increases with normal aging [18-20]. In the present study a significant beta-coefficient was observed in control cases for GFAP (Table 3), indicating an increased occurrence of astrocytes with normal aging. Although levels of microglia appear to increase slightly with aging in controls, no significant changes were observed. Probably, the statistical power of the current study is too low to observe a significant increase in microglia over age in controls. Other explanations, like differences in detection technique and markers, cannot be excluded. The previously observed increased occurrence of these markers for microglia and astrocytes with normal aging suggests that these markers could be considered as markers for senescence rather than for inflammation. This suggests the rather controversial idea that the high expression levels of these markers in younger AD patients are a marker of 'exacerbated glial senescence' at relatively young ages that could be involved in the pathogenesis of $\mathrm{AD}$. Another interpretation of this data could be that successful aging, i.e. without dementia, is associated with an increased presence and function of microglial cells, due to the increasing demand on the neuro-supportive and neuroprotective roles of microglia [21]. There are indications that microglia have a role in neurite development and it is hypothesized that microglia regulate synapse physiology [22]. The increase in 

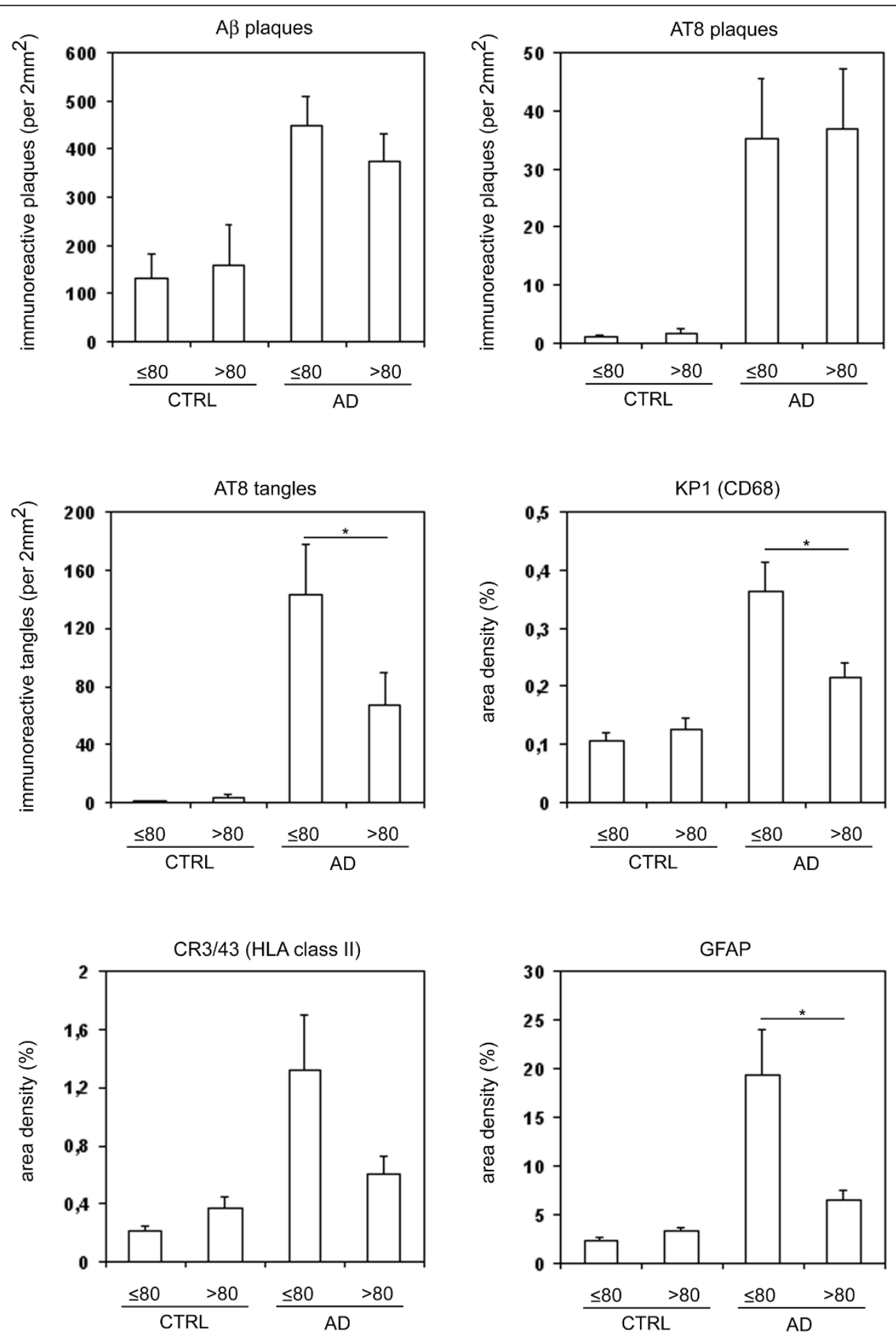

Figure 1 Relative occurrence of pathological hallmarks and neuroinflammation in controls and AD cases. Data represent the occurrence ( $Y$ axis in number per $2 \mathrm{~mm}^{2}$ ) of $A \beta$ immunoreactive plaques, AT8 immunoreactive plaques and tangles, and the relative occurrence ( $Y$ axis in percentage) of quantified KP1 (CD68), CR3/43 (HLA class II), and GFAP immunoreactivity in control (CTRL) and AD cases of 80 years and younger $(\leq 80)$ or older than 80 years $(>80)$. Data are shown as mean level \pm S.E.M.. * indicates significant difference. 

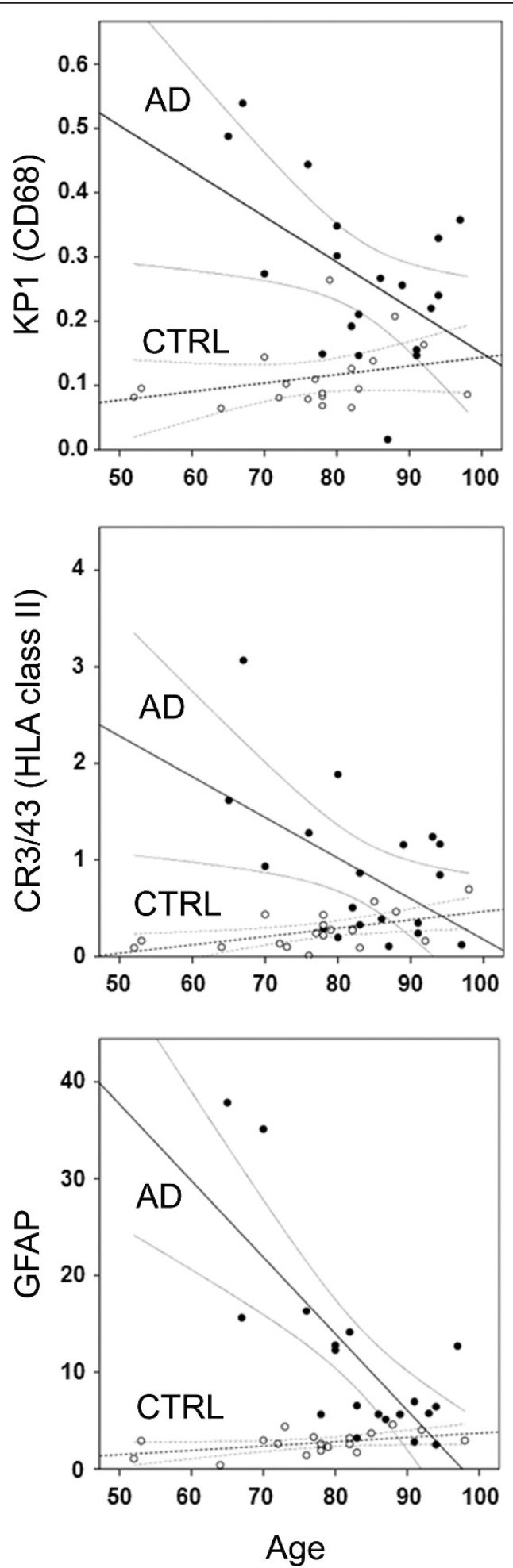

Figure 2 Neuroinflammation in relation to age in controls and AD cases. Data represent the relation between age ( $X$ axis, in years) and quantified KP1 (CD68), CR3/43 (HLA class II), and GFAP immunoreactivity ( $Y$ axis, in arbitrary units) for controls (open dots) and AD patients (closed dots). Straight lines represent regression lines, with corresponding 95\% confidence intervals.
Table 3 Beta-coefficients of the regression of the agedependent scores for $A \beta$ deposits, AT8-positive neuritic plaques, AT8-positive neurofibrillary tangles, KP1, CR3/43 and GFAP immunoreactivity for control and AD cases

\begin{tabular}{llll}
\hline \multicolumn{4}{l}{ Beta-coefficient (Standard Error) } \\
\hline Variable & CTRL & AD & p-value \\
\hline A $\beta$ deposits & $5.411(3.672)$ & $-2.611(4.845)$ & 0.193 \\
\hline AT8 NPs & $0.069(0.033)^{*}$ & $0.153(0.831)$ & 0.912 \\
\hline AT8 NFTs & $0.180(0.062)^{*}$ & $-2.416(2.247)$ & 0.212 \\
\hline KP1 & $0.001(0.001)$ & $-0.007(0.003)^{*}$ & 0.006 \\
\hline CR3/43 & $0.009(0.003)$ & $-0.042(0.017)^{*}$ & 0.003 \\
\hline GFAP & $0.044(0.020)^{*}$ & $-0.791(0.173)^{*}$ & 0.000 \\
\hline
\end{tabular}

NPs, neuritic plaques; NFTs, neurofibrillary tangles; CTRL, control case; $A D$, Alzheimer's disease cases. P-value indicates difference between regressions of the $A D$ and control groups. * indicates significant beta-coefficient.

microglia in young $\mathrm{AD}$ cases might reflect a regenerative response [23], which might become less effective with increasing age in $\mathrm{AD}$. The connection between microglia and neuronal function is reflected in the significantly higher number of neurofibrillary tangles in AD patients younger than 80 years compared to older AD patients (Figure 1).

The observed decrease in neuroinflammation in the $\mathrm{AD}$ group could reflect a selective loss of glial cells with the aging process. It has been observed that microglial cells in aged human brain are dystrophic, showing morphological features indicative of senescence and degeneration, such as cytoplasmic processes [24]. Association of senescent microglia with tau pathology in $\mathrm{AD}$ may be interpreted as an indirect sign of the age-dependent failing of the neurosupportive and neuroprotective roles of microglia that contribute to the neurodegenerative process in $\mathrm{AD}$ $[12,21,24]$. Determination of microglia senescence requires a more detailed qualitative morphological assessment. The quantification method used in this study did not assess microglial morphology, which is required for this phenotypic characterization. In addition, the markers used in the current study are those generally used for the detection of microglia and are not indicative of microglial activation state [25-28], thereby not reflecting the dual role of microglia in the inflammatory response in AD [29,30]. Future studies are needed to address activation state and phenotypic morphology, as well as selective loss of glial cells in $\mathrm{AD}$ in relation to aging.

Inheritance of APOE4, the gene that encodes apolipoprotein E4, is a major risk factor for late onset AD. The incidence of the APOE4 allele in the general population is 20-25\%, whereas the incidence in patients with AD rises to $50-65 \%$. The presence of at least one APOE4 allele has been associated with earlier age at onset [17]. The hypothesised frequency distribution of the association between age at onset and the presence of APOE4 would therefore suggest that the occurrence of APOE4 decreases 
with age in people with $\mathrm{AD}$ [31]. The apoE protein is an immunomodulatory protein that affects both innate and adaptive immune responses. Microglia derived form targeted replacement mice carrying two copies of the APOE4 allele show a pro-inflammatory phenotype compared to microglia derived from mice carrying two copies of the APOE3 allele [16]. In the present study the incidence of APOE 4 in AD cases of 80 years and younger was $57 \%$, while the incidence of APOE4 in AD cases older than 80 years was much lower, at 29\% (Table 1). This decreased frequency of APOE4 alleles could explain the difference in occurrence of neuroinflammation between young and old AD patients, and supports the hypothesis that a proinflammatory genetic profile contributes to an earlier onset of AD.

Inflammation has been advanced as one of the underlying mechanisms that drives AD pathology; however, clinical trials with anti-inflammatory drugs have generated inconclusive results [32]. The timing of anti-inflammatory treatment is crucial since clinical studies have indicated that inflammation occurs in early stages of $\mathrm{AD}$, perhaps even before exceeding the clinical detection threshold $[9,10]$. Data from the current study would imply that age itself could be an important factor for the response to therapy since the occurrence of inflammation in AD dementia decreases with increasing age of death. In addition, we show that the occurrence of microglia and astrocytes in AD and non-demented cases starts to overlap between post-mortem ages of 80 and 90 years. This data is in line with earlier studies which report considerable overlap in the severity of neurofibrillary tangle and neuritic plaque pathology between older patients with $\mathrm{AD}$ dementia and non-demented control cases $[33,34]$. The study of Savva et al. implies that the diagnostic value of biomarkers related to the underlying biological process leading to accumulation of tangles or plaques is much more prominent in the relatively young compared with the oldest patients, and indicates that the pathological substrate that causes dementia is heterogeneous and age-dependent [33]. The age-dependent relationship between the underlying biology and clinical phenotype of AD has implications for the interpretation of biomarkers and treatment of the disease. Data from the current study implicates the importance of considering age when interpreting the results of studies on inflammatory biomarkers for AD.

\section{Acknowledgements}

This study was financially supported by the Internationale Stichting Alzheimer Onderzoek (ISAO).

\section{Author details}

'Department of Pathology, VU University Medical Center, P.O. Box 7057, 1007 MB Amsterdam, The Netherlands. ${ }^{2}$ Department of Psychiatry, VU University Medical Center, Valeriusplein 9, 1075 BG Amsterdam, The Netherlands. ${ }^{3}$ Department of Neurology, Academic Medical Center,
University of Amsterdam, P.O. Box 22660, 1100 DD Amsterdam, The Netherlands.

\section{Authors' contributions}

JJMH, PE and WAVG designed the study. JJMH coordinated the study and was responsible for writing the manuscript. ESvH carried out most of the lab work and analyzed the data. PE and WAvG participated in writing the manuscript. AJMR was responsible for the autopsy material and neuropathological evaluation. All authors read and approved the final manuscript.

\section{Competing interests}

The authors declare that they have no competing interests.

Received: 11 October 2011 Accepted: 7 December 2011

Published: 7 December 2011

\section{References}

1. Akiyama H, Barger S, Barnum S, Bradt B, Bauer J, Cole GM, Cooper NR, Eikelenboom P, Emmerling M, Fiebich BL, et al: Inflammation and Alzheimer's disease. Neurobiol Aging 2000, 21:383-421.

2. Arends YM, Duyckaerts C, Rozemuller JM, Eikelenboom P, Hauw JJ: Microglia, amyloid and dementia in alzheimer disease. A correlative study. Neurobiol Aging 2000, 21:39-47.

3. Vehmas AK, Kawas CH, Stewart WF, Troncoso JC: Immune reactive cells in senile plaques and cognitive decline in Alzheimer's disease. Neurobiol Aging 2003, 24:321-31.

4. Hoozemans JJ, Van Haastert ES, Veerhuis R, Arendt T, Scheper W, Eikelenboom P, Rozemuller AJ: Maximal COX-2 and ppRb expression in neurons occurs during early Braak stages prior to the maximal activation of astrocytes and microglia in Alzheimer's disease. $J$ Neuroinflammation 2005, 2:27.

5. Harold D, Abraham R, Hollingworth P, Sims R, Gerrish A, Hamshere ML, Pahwa JS, Moskvina V, Dowzell K, Williams A, et al: Genome-wide association study identifies variants at CLU and PICALM associated with Alzheimer's disease. Nat Genet 2009, 41:1088-1093.

6. Lambert JC, Heath S, Even G, Campion D, Sleegers K, Hiltunen M, Combarros O, Zelenika D, Bullido MJ, Tavernier B, et al: Genome-wide association study identifies variants at CLU and CR1 associated with Alzheimer's disease. Nat Genet 2009, 41:1094-1099.

7. Mrak RE, Griffin WST: Glia and their cytokines in progression of neurodegeneration. Neurobiology of Aging 2005, 26:349-354.

8. Hoozemans JJ, Veerhuis R, Rozemuller JM, Eikelenboom P: Soothing the inflamed brain: effect of non-steroidal anti-inflammatory drugs on Alzheimer's disease pathology. CNS Neurol Disord Drug Targets 2011, 10:57-67.

9. Cagnin A, Brooks DJ, Kennedy AM, Gunn RN, Myers R, Turkheimer FE, Jones T, Banati RB: In-vivo measurement of activated microglia in dementia. Lancet 2001, 358:461-7.

10. Okello A, Edison P, Archer HA, Turkheimer FE, Kennedy J, Bullock R, Walker Z, Kennedy A, Fox N, Rossor M, et al: Microglial activation and amyloid deposition in mild cognitive impairment: a PET study. Neurology 2009, 72:56-62.

11. Franceschi C, Bonafe M: Centenarians as a model for healthy aging. Biochem Soc Trans 2003, 31:457-461

12. Streit WJ, Braak H, Xue QS, Bechmann I: Dystrophic (senescent) rather than activated microglial cells are associated with tau pathology and likely precede neurodegeneration in Alzheimer's disease. Acta Neuropathol 2009, 118:475-485.

13. Reisberg B, Ferris SH, de Leon MJ, Crook T: The Global Deterioration Scale for assessment of primary degenerative dementia. Am J Psychiatry 1982, 139:1136-9.

14. Yamaguchi H, Haga C, Hirai S, Nakazato Y, Kosaka K: Distinctive, rapid, and easy labeling of diffuse plaques in the Alzheimer brains by a new methenamine silver stain. Acta Neuropathol (Berl) 1990, 79:569-72.

15. Braak H, Braak E: Neuropathological stageing of Alzheimer-related changes. Acta Neuropathol (Berl) 1991, 82:239-259.

16. Vitek MP, Brown CM, Colton CA: APOE genotype-specific differences in the innate immune response. Neurobiol Aging 2009, 30:1350-1360.

17. Meyer MR, Tschanz JT, Norton MC, Welsh-Bohmer KA, Steffens DC, Wyse BW, Breitner JC: APOE genotype predicts when-not whether-one is predisposed to develop Alzheimer disease. Nat Genet 1998, 19:321-322. 
18. Sheng JG, Mrak RE, Griffin WS: Enlarged and phagocytic, but not primed, interleukin-1 alpha-immunoreactive microglia increase with age in normal human brain. Acta Neuropathol 1998, 95:229-234.

19. Schuitemaker A, van der Doef TF, Boellaard R, van der Flier WM, Yaqub M, Windhorst AD, Barkhof F, Jonker C, Kloet RW, Lammertsma AA, et al: Microglial activation in healthy aging. Neurobiol Aging 2010

20. Beach TG, Walker R, McGeer EG: Patterns of gliosis in Alzheimer's disease and aging cerebrum. Glia 1989, 2:420-436.

21. Streit WJ: Microglia and neuroprotection: implications for Alzheimer's disease. Brain Res Brain Res Rev 2005, 48:234-239.

22. Pont-Lezica L, Bechade C, Belarif-Cantaut Y, Pascual O, Bessis A: Physiological roles of microglia during development. J Neurochem 2011, 119:901-908.

23. Hoozemans JJ, Veerhuis R, Rozemuller JM, Eikelenboom P: Neuroinflammation and regeneration in the early stages of Alzheimer's disease pathology. Int J Dev Neurosci 2006, 24:157-165.

24. Streit WJ, Sammons NW, Kuhns AJ, Sparks DL: Dystrophic microglia in the aging human brain. Glia 2004, 45:208-212.

25. Ramprasad MP, Terpstra V, Kondratenko N, Quehenberger O, Steinberg D: Cell surface expression of mouse macrosialin and human CD68 and their role as macrophage receptors for oxidized low density lipoprotein. Proc Natl Acad Sci USA 1996, 93:14833-14838.

26. Deininger MH, Pater S, Strik H, Meyermann R: Macrophage/microglial cell subpopulations in glioblastoma multiforme relapses are differentially altered by radiochemotherapy. J Neurooncol 2001, 55:141-147.

27. Caffo M, Caruso G, Germano A, Galatioto S, Meli F, Tomasello F: CD68 and CR3/43 immunohistochemical expression in secretory meningiomas. Neurosurgery 2005, 57:551-557.

28. Kim SU, de Vellis J: Microglia in health and disease. J Neurosci Res 2005, 81:302-313.

29. Colton CA, Wilcock DM: Assessing activation states in microglia. CNS Neurol Disord Drug Targets 2010, 9:174-191.

30. Wyss-Coray T, Mucke L: Inflammation in neurodegenerative disease-a double-edged sword. Neuron 2002, 35:419-32.

31. van der Flier WM, Pijnenburg YA, Fox NC, Scheltens P: Early-onset versus late-onset Alzheimer's disease: the case of the missing APOE varepsilon4 allele. Lancet Neurol 2011, 10:280-288.

32. van Gool WA, Aisen PS, Eikelenboom P: Anti-inflammatory therapy in Alzheimer's disease: is hope still alive? I Neurol 2003, 250:788-92

33. Savva GM, Wharton SB, Ince PG, Forster G, Matthews FE, Brayne C: Age, neuropathology, and dementia. N Engl I Med 2009, 360:2302-2309.

34. van Gool WA, Eikelenboom P: The two faces of Alzheimer's disease. J Neurol 2000, 247:500-505

doi:10.1186/1742-2094-8-17

Cite this article as: Hoozemans et al:: Neuroinflammation in Alzheimer's disease wanes with age. Journal of Neuroinflammation 2011 8:171.

\section{Submit your next manuscript to BioMed Central and take full advantage of:}

- Convenient online submission

- Thorough peer review

- No space constraints or color figure charges

- Immediate publication on acceptance

- Inclusion in PubMed, CAS, Scopus and Google Scholar

- Research which is freely available for redistribution

Submit your manuscript at www.biomedcentral.com/submit
C Biomed Central 